\title{
Ultraslow Relaxation Process of Static Light Scattering Intensity by Boron Oxide above the Glass Transition Temperature
}

\author{
N. A. Bokov \\ Institute of Silicate Chemistry, Russian Academy of Sciences, St. Petersburg, Russia \\ Email: bokov@isc1.nw.ru
}

Received 1 April 2015; accepted 24 June 2015; published 1 July 2015

\begin{abstract}
The data describing of the light scattering intensity relaxation above the glass transition temperature by boron oxide obtained by using of the temperature jump methods are presented. It is found that the stabilization of the glass at $220^{\circ} \mathrm{C}$ resulted in increasing of the anisotropic intensity at the same time the isotropic intensity is not practically unchangeable. It is shown that after the temperature jump to $330^{\circ} \mathrm{C}$ the intensity is characterized by the formation of a maximum. This effect is in compliance with the results obtained for the other oxide glasses. It is established that characteristic time of the relaxation of the maximum height is about two orders of magnitude more than the structural relaxation time for this temperature.
\end{abstract}

\section{Keywords}

\section{Static Light Scattering, Glass Transition, Relaxation Time, Boron Oxide}

\section{Introduction}

It is known [1] [2] that the change of the visible light scattering (VLS) intensity by boron oxide in the glass transition region demonstrates some specific particularities, which mismatch with the universal regularities observed for the other oxide glasses [3]-[5]. A new feature of the VLS intensity relaxation by boron oxide is found in the recent work [6]. The obtained data demonstrate that the relaxation process of the integral VLS intensity at the temperature $330^{\circ} \mathrm{C}$ after the heating of the sample from $220^{\circ} \mathrm{C}$ is characterized by the relaxation time equal about 50 min which far exceeded the expected times for the structural relaxation at this temperature.

The aim of the present work is to study in details this unusual feature of the relaxation process in boron oxide using the method of the temperature jumps.

\section{Experimental}

Measurements of the VLS intensity is conducted using a high temperature light diffractometer manufactured in the laboratory described more detail in [7]. A diode-pumped solid-state (DPSS) laser with a radiation power of $100 \mathrm{~mW}$ and light wavelength $\lambda=532 \mathrm{~nm}$ is used as the source of a primary radiation. The values obtained ex- 
perimentally are the polarized, $V_{v}$, and depolarized, $H_{v}$, components of the VLS intensity, where the index denotes the polarization status of the incident beam and the capital letters stand for the orientation of polarizer before the detector.

In any liquid, the above-mentioned components are connected with the Rayleigh ratio measured at the scattering angle of $90^{\circ}$. This relation-ship is presented by the equations $R_{\text {is }}=V_{v}-4 / 3 H_{v}$ and $R_{a n}=7 / 3 H_{v}$, which permit one to separate these scattering coefficients [8].

The intensity of the polarized $\mathrm{V}_{\mathrm{v}}$ and depolarized $\mathrm{H}_{\mathrm{v}}$ components of the VLS intensity recorded at scattering angle of $90^{\circ}$ is presented in benzene units; i.e., it is normalized to the benzene isotropic scattering.

The experimental procedure is carried out according to the following scheme:

i) A sample is stabilized at the temperature $220^{\circ} \mathrm{C}$ during the different time (stabilization time); and

ii) A sample temperature is abruptly increased up to $330^{\circ} \mathrm{C}$ at which the VLS intensity is measured as a function of time; after that

iii) A sample is cooled to the stabilization temperature for the starting of a new cycle of the measurement.

The samples of boron oxide that were used in [1] [3] and stored in a hermetically sealed cell filled with diffusion oil were selected for conducting the experiments. The samples have a shape of a right angle cylinder with a diameter of $2.7 \mathrm{~cm}$ and height of $1.1 \mathrm{~cm}$. The samples were carefully polished just before the procedure to remove the formed plaque. The volume of the furnace is filled with dry argon in order to prevent the effect of humidity on the polished surface of the sample. First, confirm that you have the correct template for your paper size. This template has been tailored for output on the custom paper size $(21 \mathrm{~cm} * 28.5 \mathrm{~cm})$.

\section{Results}

Figure 1 demonstrates the experimental results corresponding to the dependences on stabilization time for the polarized, $\mathrm{V}_{\mathrm{v}}$, and depolarized, $\mathrm{H}_{\mathrm{v}}$, components of the VLS intensity at the stabilization temperature $220^{\circ} \mathrm{C}$.

As follows from the data on Figure 1, the intensities of both components increase with the increasing of stabilization time. At the same time Figure 1 demonstrates the results of the calculations of the coefficient of isotropic light scattering $R_{\text {is }}$. It is obvious that the coefficient of isotropic light scattering $R_{\text {is }}$ is almost constant during of the process of low temperature annealing. This result indicates that the recorded variations in the intensities of the $V_{v}$ and $H_{v}$ components are probably caused only by the increase of the anisotropic scattering $R_{a n}$. At present time no rational explanation has been suggested for the observed peculiarities, and, hence, further detailed investigation is required.

As mentioned above after each stabilization time at the temperature $220^{\circ} \mathrm{C}$ the sample temperature is abruptly increased up to the value $330^{\circ} \mathrm{C}$ at which the VLS intensity is measured as a function of time. Figure 2 illustrates the time dependences of the polarized, $\mathrm{V}_{\mathrm{v}}$, intensity at the temperature $330^{\circ} \mathrm{C}$ after temperature jump

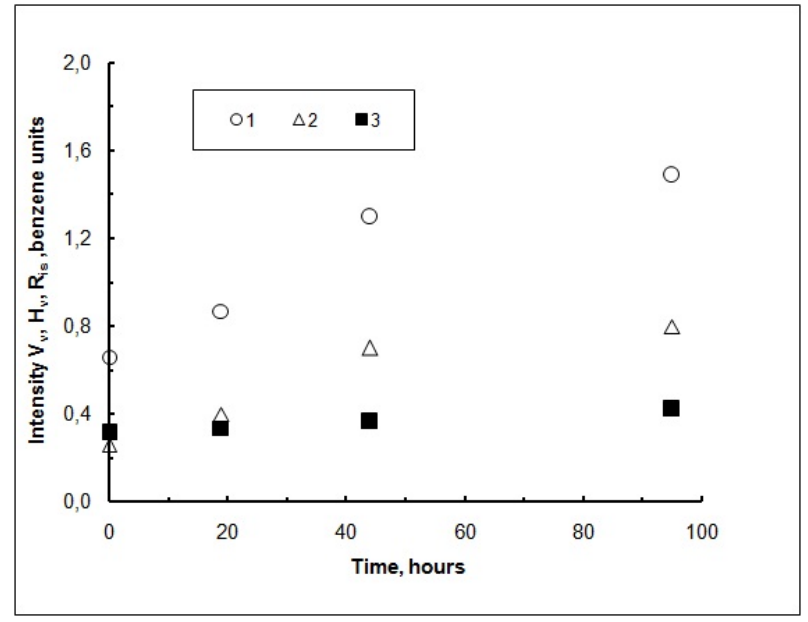

Figure 1. Dependences of the polarized $\mathrm{V}_{\mathrm{v}}(1)$ and depolarized $H_{v}(2)$ components of the VLS intensity and the coefficient of isotropic scattering $\mathrm{R}_{\mathrm{is}}(3)$ on the stabilization time at the temperature $220^{\circ} \mathrm{C}$. 


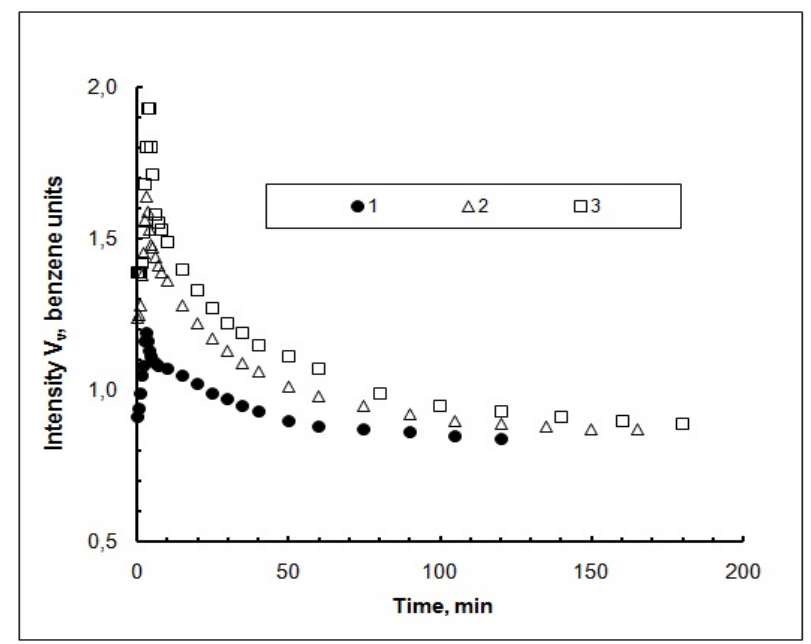

Figure 2. Time dependences of the polarized $\mathrm{V}_{\mathrm{v}}$ components of the VLS intensity at the temperature $330^{\circ} \mathrm{C}$ after temperature jump from $220^{\circ} \mathrm{C}$. Stabilization time for the sample at the temperature $220^{\circ} \mathrm{C}$ were (1) 19 , (2) 44 , (3) $95 \mathrm{~h}$.

from the value $220^{\circ} \mathrm{C}$ for the following selected stabilization time: 19,44 and 95 hours.

Figure 2 shows that as usual for oxide glasses under isothermal condition, the time dependence of $V_{v}$ intensity is characterized by a peak. Moreover, an increase of the stabilization time leads to an increase of the peak height and the total duration of the relaxation process [3]-[5]. Finally, one can see from Figure 2 that the unusual characteristic of a maximum observed is to significant asymmetry of its shape. It is obviously that the right side of the maximum is too much long as a function of time.

Figure 3 more evidently demonstrates the marked characteristic of the registered peak using logarithmic scale of the time axis. As follows from Figure 3 the time dependence of the polarized, $V_{v}$, component may be considered consisting from the two parts: the first part corresponds to the time smaller $10 \mathrm{~min}$, where the maximum height is observed, and the second part relates with the times more $10 \mathrm{~min}$. It may be proposed that the first part connects with the universal effect observed earlier in the all oxide glasses [3]-[5]. As it is found that the second part (right side of a maximum) is satisfactorily described by the usual exponential dependence in the form $V_{v}(t)$ $=\mathrm{A} \exp (-\mathrm{t} / \tau)+\mathrm{B}$, where $\mathrm{A}$ and $\mathrm{B}$ are constants, $\mathrm{t}$ is time, $\tau$ is relaxation time.

Figure 4 represents a graph of the quantity $\ln \left(V_{v}-B\right)$ as a function of time constructed according to the experimental data. As follows from Figure 4 the obtained regularities are satisfactorily described by the linear dependences for the each preliminary stabilization time at the temperature $220^{\circ} \mathrm{C}: 19,44$ and 95 hours. The calculated values of the relaxation time, $\tau$, were approximately 30,35 and 45 min, correspondingly. Very large characteristic times of the observed process of the intensity decreasing are the important feature of the obtained results.

It should be noted that the obtained values of the relaxation time, $\tau$, exceed significantly the predicted time of the structural relaxation at the temperature $330^{\circ} \mathrm{C}$, which is estimated in accordance with the Maxwell equation $\tau$ $=\eta / \mathrm{G}_{\infty}$, where $\tau$ is the relaxation time, $\eta$ is the shear viscosity, and $\mathrm{G}_{\infty}$ is the instantaneous shear module [6]. In accordance with this approach the relaxation time $\tau$ is about $2-3$ min.

It should be noted in conclusion that after the stationary intensity is achieved during the stabilization at the temperature $330^{\circ} \mathrm{C}$, the sample is cooled to the temperature of preliminary annealing at the temperature $220^{\circ} \mathrm{C}$. Figure 5 represents the temperature dependences of the intensity recorded at the cooling rate of $4 \% \mathrm{~min}$. The data illustrate that the recorded variations in the intensities are in agreement within the experimental error of $5 \%$. The intensities attained during the cooling of the sample to the temperature $220^{\circ} \mathrm{C}$ correspond to approximately the identical value. The result obtained indicates the increase of the polarized component $\mathrm{V}_{\mathrm{v}}$ intensity during of the low temperature annealing of the glass at the temperature $220^{\circ} \mathrm{C}$ (Figure 1).

\section{Discussion}

It is known [9]-[11] that at the glass transition of polymers result in the following unusual features observed in a 


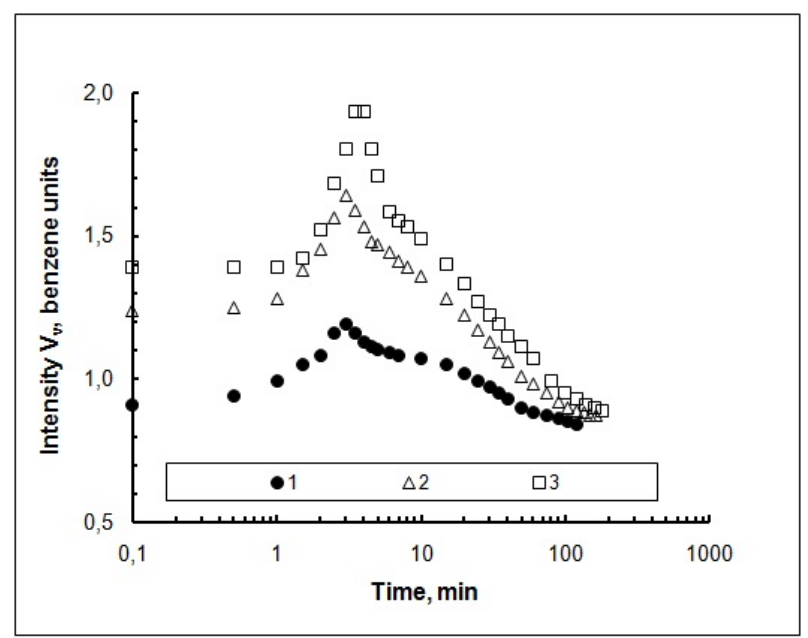

Figure 3. Time dependences of the polarized $\mathrm{V}_{\mathrm{v}}$ components of the VLS intensity at $330^{\circ} \mathrm{C}$ after temperature jump from $220^{\circ} \mathrm{C}$. Stabilization time for the sample at temperature $220^{\circ} \mathrm{C}$ were (1) 19, (2) 44, (3) $95 \mathrm{~h}$.

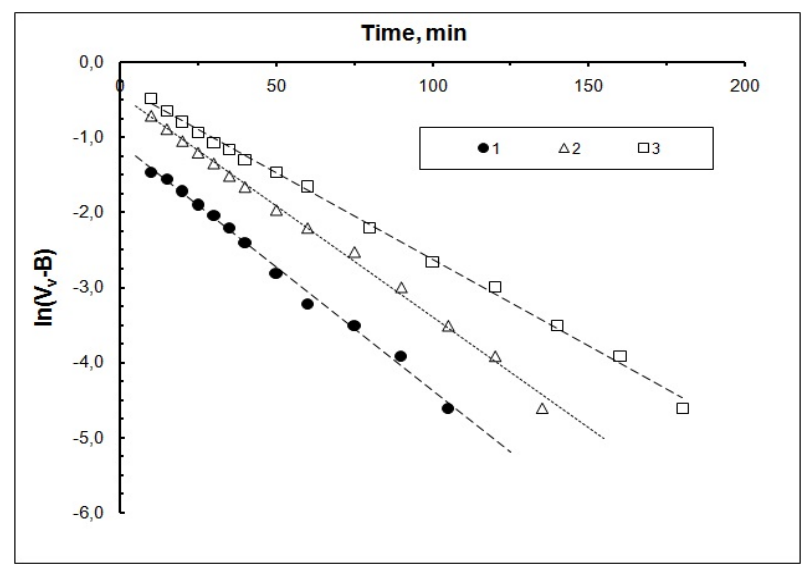

Figure 4. Dependence of the quantity $\ln \left(\mathrm{V}_{\mathrm{v}}-\mathrm{B}\right)$ at $330^{\circ} \mathrm{C}$ after temperature jump from the temperature $220^{\circ} \mathrm{C}$. Stabilization time for the sample at the temperature $220^{\circ} \mathrm{C}$ were (1) 19 , (2) 44, (3) $95 \mathrm{~h}$.

light scattering experiment, which are not described by the existing theories: the existence of the ultra-slow mode with characteristic times considerably higher than the times of $\alpha$-relaxation process; the spread of the halo from the light beam passed through the sample above their glass transition temperature; the appearance a speckle pattern in the halo. Based on the obtained experimental data, a theory of the structural, kinetic, and dynamic features of the Fischer cluster is put forward. This theory is based on the idea of heterophase fluctuations of glass-forming liquids.

It should be noted that the spatial distribution of scattered intensity is not studied in the present investigation. However, a visual observation demonstrates that the development of a maximum recorded at an angle of $90^{\circ}$ is accompanied by the appearance of a very intense halo in the neighborhood of the primary light beam passed through the glass sample. This effect indicates a considerable increase in the intensity of scattering radiation in small-angle range. This phenomenon can be caused by large-scale structural rearrangements.

The considered features point out on the possible correlation of the phenomena observed for the polymers and oxide glasses. However, it should be mentioned that the increasing of the VLS intensity in small-angle range may be connected with the effect of nonlinear coupling of the laser radiation with the unstable glass structure as an VLS intensity peak is observed at scattered angle $90^{\circ}$ [12]. 


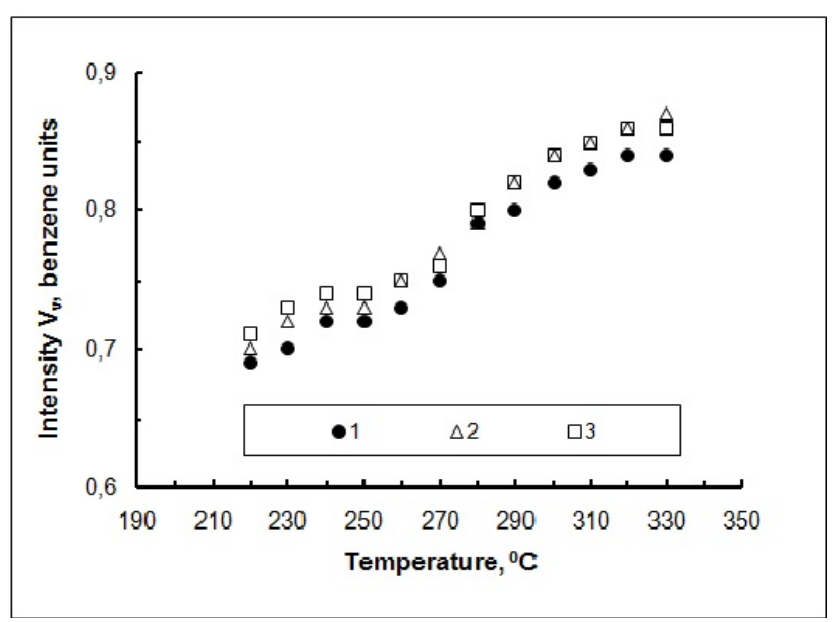

Figure 5. Temperature dependence of the polarized $\mathrm{V}_{\mathrm{v}}$ component of the VLS intensity during the cooling of boron oxide recorded for a sample preliminary annealed at the temperature $220^{\circ} \mathrm{C}$ for (1) 19 , (2) 44, (3) $95 \mathrm{~h}$.

Generally based on the fact that the relaxation time has extremely long duration it may be assumed that the main physical reason of this phenomenon connected with the thermal diffusion process.

\section{Conclusion}

The results obtained in present study using the temperature jump technique confirm the unusual feature of the existence of the ultra slow relaxation in boron oxide found earlier. It should be underlined that this effect is observed at the temperature above glass transition range. At present time the reason of this phenomenon has not been yet clarified and further studies could yield new insights.

\section{Acknowledgements}

The work was supported by grant no. 13-03-00718 of the Russian Fund for Basic Research.

\section{References}

[1] Bokov, N.A. and Andreev, N.S. (1989) Light Scattering by Boron Oxide in the Glass Transition Range. Fizika Khimia Stekla, 15, 424-427.

[2] Bokov, N.A. (1994) Light Scattering Studies of Glasses in the Glass Transition Region. Journal of Non-Crystalline Solids, 177, 74-80. http://dx.doi.org/10.1016/0022-3093(94)90516-9

[3] Bokov, N.A. and Andreev, N.S. (1999) Light Scattering by Sodium Germanate Glass after Temperature Jumps in the Glass Transition Range. Glass Physics Chemistry, 25, 125-129.

[4] Bokov, N.A. and Andreev, N.S. (2004) Specific Features of Light Scattering by Oxide Glasses in the Glass Transition Range. Glass Physics Chemistry, 30, 6-13. http://dx.doi.org/10.1023/B:GPAC.0000016392.99186.45

[5] Bokov, N.A. (2006) Influence of the Thermal Prehistory of the Silicate Glass on the Intensity of Light Scattering after Temperature Jumps in the Glass Transition Range. Glass Physics Chemistry, 33, 475-480. http://dx.doi.org/10.1134/S1087659607050082

[6] Bokov, N.A. (2015) Light Scattering Investigation of a Slow Relaxation Process of Boric Oxide in the Glass Transition Region. Glass Physics Chemistry, 41, 254-257. http://dx.doi.org/10.1134/S1087659615010071

[7] Bokov, N.A. (1992) Molecular Scattering of Light by Alkali Borate Melts and Glasses. Ph.D. Thesis, Leningrad State University, Leningrad.

[8] Vuks, M.F. (1977) Light Scattering in Gases, Liquids, and Solutions. Leningrad State University, Leningrad.

[9] Fisher, E.W. (1993) Light Scattering and Dielectric Studies on Glass Forming Liquids. Physica A, 201, 183-206. http://dx.doi.org/10.1016/0378-4371(93)90416-2

[10] Fischer, E.W., Bakai, A., Patkowski, A., Steffen, W. and Reinhardt, L. (2002) Heterophase Fluctuations in Super- 
cooled Liquids and Polymers. Journal of Non-Crystalline Solids, 307-310, 584-601. http://dx.doi.org/10.1016/S0022-3093(02)01510-7

[11] Bakai, A.S. and Fisher, E.W. (2004) Nature of Long-Range Correlations of Density Fluctuations in Glass-Forming Liquids. Journal of Chemical Physics, 120, 5235-5252. http://dx.doi.org/10.1063/1.1648300

[12] Bokov, N.A. and Andreev, N.S. (2001) Optical Modulation of the Refractive Index in the Glass Transition Range. Glass Physics Chemistry, 27, 547-550. http://dx.doi.org/10.1023/A:1013254410346 